\section{Comments on "Time Optimal Feedback Control for Small Disturbances"}

\section{G. J. OLSDER}

Abstract-The problem in the above-mentioned short paper ${ }^{1}$ is almost Identical to the one considered earlier by the present author and, in fact, a special version of it. This note elaborates on this close relation between the results of the two papers.

The purpose of this note is to point out the close relationship between Meeker's paper ${ }^{1}$ and [1]. The latter publication is a concise version of [2]. Actually, the main results presented by Meeker (Section IV) are by and large a special case of the general theory treated in [1], although the terminology and approaches are different.

The starting point in both Meeker and [1] is the multiple input system

$$
\dot{x}=A x+B u
$$

where $x$ is an $n$-vector and $u$ is an $r$-vector. The columns of $B$, to be denoted by $b^{1}, \cdots, b r$, are supposed to be linearly independent. The components of $u$, to be denoted by $u_{1}, \cdots, u_{r}$, must be chosen in such a way that the system (1) is time-optimally steered from a given initial point $x_{0} \in R^{n}$ to the origin in $R^{n}$, subject to $\left|u_{i}\right| \leqslant 1, i=1, \cdots, r$. In both papers $x_{0}$ must be sufficiently close to the origin, and the central problem deals with the number of switches per control component of the time optimal control and the signs (either +1 of -1 ) of the control components at the initial time.

In Meeker, ${ }^{1}$ the concept of a "minimally controllable system" is introduced for which the definition is as follows: (1) is minimally controllable if, for all $x_{0}$ in a sufficiently small neighborhood of the origin, a corresponding time optimal control exists which has at most $n-1$ switches (i.e., over all control components together). Only for systems of size $n=3, r=2$ is a necessary and sufficient condition given for (1) to be minimally controllable. That condition is

$$
\operatorname{det}\left[b^{1}, b^{2}, A b^{i}\right] \neq 0, \quad i=1,2 .
$$

In [1] the class of $L^{*}$-systems is introduced: (1) is an $L^{*}$-system if certain conditions with respect to the matrices $A$ and $B$ are fulfilled. Both the class of minimally controllable systems and $L^{*}$-systems are generic. It can be proven that if one confines the class of minimally controllable systems to that class of systems for which, for all $x_{0}$ in a sufficiently small neighborhood of the origin, the time optimal control is unique, then one obtains the $L^{*}$-class. From now on the initial condition will be written as $\epsilon x_{0}$ where $\left\|x_{0}\right\|=1,\|\cdot\|$ being the Euclidian norm and $\epsilon$ a small positive number. In the following, $p$ is the smallest integer greater than or equal to $n / r$.

The main theorem of [1] states that if: i) system (1) is an $L^{*}$-system, ii) $x_{0}$ does not lie within a finite number of given hyperplanes, iii) $\epsilon$ is sufficiently small (which may depend on the direction of $x_{0}$ ), then

a) the time optimal control which steers (1) from $e x_{0}$ to the origin is unique and each control component has either $p-1$ or $p$ switches. The total number of switches equals $n-1$,

b) explicit formulas exist and are given in [1] which determine the starting signs of the control components in terms of $A, B$, and $x_{0}$,

c) the switching times and final time are analytic functions of $\sqrt[P]{\epsilon}$.

If we confine ourselves to systems for which $n=3, r=2$, as done by Meeker, ${ }^{1}[1]$ yields the following results. $L^{*}$-systems are exactly those systems for which (2) holds. Hence, for $n=3, r=2$, the classes of minimally controllable systems and $L^{*}$-systems are identical. The $x_{0}$ excluded in the main theorem of [1] are those for which

$$
\operatorname{det}\left[B, x_{0}\right]=0 .
$$

Manuscript received June 29, 1979.

The author is with the Department of Applied Mathematics, Twente University of Technology, Enschede, The Netherlands, on leave at Haryard University, Cambridge, MA 02138 .

'L. D. Meeker, IEEE Trans. Automat. Contr., vol. AC-23, pp. 1095-1099, Dec. 1978.
The vector $\psi_{0} \in R^{3}$ is uniquely defined by

$$
\left\|\psi_{0}\right\|=1, \psi_{0} \perp b^{i}, \quad i=1,2,\left(\psi_{0}, x_{0}\right)<0
$$

where $(\cdot, \cdot)$ denotes the inner product. For $\varepsilon$ sufficiently small, the starting signs of the optimal $u_{1}(t)$ and $u_{2}(t)$ are

$$
s_{i}=\operatorname{sgn}\left(\psi_{0} A b^{i}\right), \quad i=1,2 \text {, respectively. }
$$

Because only $L^{*}$-systems are considered and $x_{0}$ which do not satisfy (3), the sgn relations in (4) are well defined. Both the optimal $u_{1}(t)$ and $u_{2}(t)$ have one switch, to be denoted by $t_{1}$ and $t_{2}$, respectively.

A cellular decomposition of all $x_{0}$ on the unit ball $\left\|x_{0}\right\|=1$ can be constructed; a cell is defined as all $x_{0}$ for which $s_{1}$ is constant and $s_{2}$ is constant. This induces a honeycomb-like structure on the unit ball, which is closely related to the cellular decomposition in Meeker.'

The switching times $t_{i}$ and the final time $T$ are analytical functions of $\sqrt{\epsilon}:$

$$
t_{i}=\sum_{j=1}^{\infty} \alpha_{i j}(\sqrt{\epsilon})^{j}, \quad i=1,2, \quad T=\sum_{j=1}^{\infty} \beta_{j}(\sqrt{\epsilon})^{j} .
$$

The coefficients $\alpha_{i j}$ and $\beta_{j}$ satisfy linear relations which are given in [1] for systems of general size $n, r$. For $(n=3, r=2)$ systems, the first coefficients are

$$
\beta_{1}=2 \sqrt{\left|\frac{\operatorname{det}\left[B, x_{0}\right]}{\operatorname{det}[M]}\right|}, \quad \alpha_{11}=\alpha_{21}=\frac{1}{2} \beta_{1}
$$

where the square matrix $M$ is defined as $M=\left[B, A\left(s_{1} b^{1}+s_{2} b^{2}\right)\right]$. For an explanation of the remarkable fact that $\alpha_{i 1} / \beta_{1}, i=1,2$, is independent of $A, B$, and $x_{0}$, see [1].

\section{REFERENCES}

[1] G. J. Olsder, "Time-optimal control of multivariable systems near the origin" $J$. Optimiz. Theory Apph, vol. 16, no. 5/6, pp. 497-517, 1975 .

[2] "On the time-optimal bang bang control of linear multivariable systems with small initial perturbations," Ph.D. dissertation, Univ. Groningen, Groningen, The Netherlands, 1971.

\section{Author's Reply ${ }^{2}$}

\section{D. MEEKER}

It is true that Dr. Olsder's papers [5], [6] (previously unknown to the author) address the same problem as that of my paper ${ }^{1}$ and, in fact, consider a less restricted system. However, as noted, the method of my paper ${ }^{1}$ has been extended to the same generality appearing in Dr. Olsder's work [1]-[3]. Furthermore, there are significant differences in the two approaches to the problem which are not adequately addressed in the comment above.

The papers [5] and [6] are focused on the determination of open-loop time-optimal control to the origin of a fixed $x^{0}$ and present yet another numerical algorithm to determine a control function to accomplish this. This procedure, which fails for some points arbitrarily near the origin, suffers also from the same weakness as the methods proposed previously - it fails to provide the information required for synthesis of a time optimal vector field (or a time-optimal feedback function).

${ }^{2}$ Manuscript received August 7, 1979.

The author is with the Department of Mathematics, University of New Hampshire, Durbam, NH 03824. 